\title{
A cost-effective melting temperature assay for the detection of single-nucleotide polymorphism in the MBL2 gene of HIV-1-infected children
}

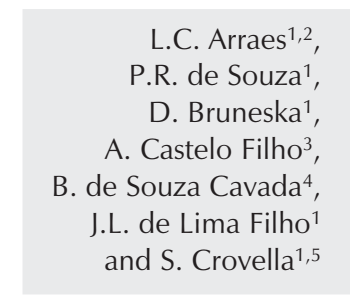

\section{Correspondence}

S. Crovella

Department of Developmental

and Reproductive Sciences

University of Trieste

Via dell'Istria 65/1

34137 Trieste

Italy

Fax: +39-040-378-5540

E-mail: crovella@burlo.trieste.it

Research supported by FINEP, LIKA-UFPE and CNPq. P.R. de Souza

and D. Bruneska are recipients

of fellowships from CNPq.

Publication supported by FAPESP.

......................

Received June 17, 2005

Accepted October 6, 2005

.....................

\author{
${ }^{1}$ Laboratório de Imunopatologia Keizo Asami, Universidade Federal de Pernambuco, \\ Recife, PE, Brasil \\ ${ }^{2}$ Instituto Materno Infantil de Pernambuco, Recife, PE, Brasil \\ ${ }^{3}$ Disciplina de Gastroenterologia, Universidade Federal de São Paulo, \\ São Paulo, SP, Brasil \\ ${ }^{4}$ Departamento de Bioquímica e Biologia Molecular, Universidade Federal do Ceará, \\ Fortaleza, CE, Brasil \\ ${ }^{5}$ Department of Developmental and Reproductive Sciences, University of Trieste, \\ Trieste, Italy
}

Abstract

We report a fast (less than $3 \mathrm{~h}$ ) and cost-effective melting temperature assay method for the detection of single-nucleotide polymorphisms in the MBL2 gene. The protocol, which is based on the Corbett Rotor Gene real time PCR platform and SYBR Green I chemistry, yielded, in the cohorts studied, sensitive (100\%) and specific (100\%) PCR amplification without the use of costly fluorophore-labeled probes or post-PCR manipulation. At the end of the PCR, the dissociation protocol included a slow heating from $60^{\circ}$ to $95^{\circ} \mathrm{C}$ in $0.2^{\circ} \mathrm{C}$ steps, with an 8-s interval between steps. Melting curve profiles were obtained using the dissociation software of the Rotor Gene-3000 apparatus. Samples were analyzed in duplicate and in different PCR runs to test the reproducibility of this technique. No supplementary data handling is required to determine the MBL2 genotype. MBL2 genotyping performed on a cohort of 164 HIV-1-positive Brazilian children and 150 healthy controls, matched for age and sex and ethnic origin, yielded reproducible results confirmed by direct sequencing of the amplicon performed in blind. The three MBL2 variants (Arg52Cys, Gly54Asp, Gly57Glu) were grouped together and called allele 0, while the combination of three wild-type alleles was called allele A. The frequency of the A/A homozygotes was significantly higher among healthy controls (0.68) than in HIV-infected children $(0.55 ; \mathrm{P}$ $=0.0234$ ) and the frequency of MBL2 0/0 homozygotes was higher among HIV-1-infected children than healthy controls $(\mathrm{P}=0.0296)$. The 0 allele was significantly more frequent among the 164 HIV-1infected children $(0.29)$ than among the 150 healthy controls $(0.18 ; \mathrm{P}$ $=0.0032$ ). Our data confirm the association between the presence of the mutated MBL2 allele (allele 0) and HIV-1 infection in perinatally exposed children. Our results are in agreement with the literature data which indicate that the presence of the allele 0 confers a relative risk of 1.37 for HIV-1 infection through vertical transmission.
Key words

- Melting temperature assay

- Real time PCR

- MBL2

- Polymorphisms

- HIV-1-positive children 
The interest in the investigation of singlenucleotide polymorphisms (SNPs) has increased significantly since the completion of the human genome project. It is widely accepted that SNPs represent the most abundant form of genetic variation between individuals. Indeed, SNPs can be considered to be powerful tools for the identification of genes involved in Mendelian, polygenic and multifactorial diseases (1). SNP analysis has been successfully used to understand the pathogenesis of several diseases. Many different techniques are currently being used for SNP detection, such as mass spectrometry (2), high-pressure liquid chromatography (3-5), microarray analysis (6), Taqman probes (7), or molecular beacons (8). All of these methods are generally costly and time consuming, facts that hinder their implementation in most laboratories, at least those more basically equipped and without the possibility of robotic automation.

Melting temperature analysis is widely accepted as a flexible and powerful screening method for the detection of mutations since it is a low-cost technique, not time consuming and can be completely automated (9). Recently Hladnik et al. (10) developed a powerful software for the interpretation of the melting profiles since the melting curves generated by the ABI 7700 Dissociation Curves software presented fluctuations in the readings, needing statistical correction in order to obtain a simple diagram for the correct interpretation of the genotype. This software adds a supplementary step to the process of SNP detection that, even if fully automated, requires some additional time after fluorescent SYBR Green I PCR.

MBL2 encodes a multichain serum lectin called mannose-binding protein (MBP), an important effector of innate immunity. MBP binds specific carbohydrate residues present on some virus, bacteria and yeast by activating the complement system on the pathogens or assisting phagocytosis by macrophages. Three missense polymorphisms at codons 52, 54, and 57 resulting in reduced serum levels of MBP have been described in the first exon of the gene and have been grouped together as “allele 0” (11). Garred et al. (11) in a cohort of homosexual men observed significantly higher frequencies of 0/0 homozygotes in HIV-1-infected patients when compared with high-risk controls. In their study, Garred et al. (11) also demonstrated a correlation between MBL2 allelic variants and a more rapid progression to death in seroconverters.

Polymorphisms of the MBL2 gene have also been associated with increased susceptibility to HIV-1 infection in Brazilian children (12) and with accelerated disease progression in HIV-1-infected Italian children (13) born to seropositive mothers.

In the present report, we describe a melting temperature assay protocol for cost-effective MBL2 SNP detection in a cohort of HIV-1-infected Brazilian children from Recife (PE, Brazil) with the aim of validating an SNP detection protocol suitable for simply equipped laboratories.

Peripheral blood was collected from 164 HIV-1-positive children at the immunological day hospital of Instituto Materno Infantil de Pernambuco. Written informed consent was obtained from the persons responsible for the patients and the study was approved by the Ethics Committee of the Institution. DNA was extracted from peripheral blood using the GenomePrep extraction kit (Amersham-Pharmacia, Buckinghamshire, UK) according to manufacturer instructions. One hundred and fifty healthy Brazilian subjects, matched for age and sex, of the same ethnic origin as the HIV-1-positive patients were enrolled as controls and written informed consent to participate in the study was obtained from the persons responsible.

MBL2 SNP genotyping was performed using the following primers designed with the Primer Express 1.5 software (Applied Biosystems, Foster City, CA, USA): forward primer 5'-AGGCATCAACGGCTT 
CCCA-3' and reverse primer 5'-CAGAA CAGCCCAACACGTACCT-3'.

The expected amplicon length is $90 \mathrm{bp}$ and its theoretical melting temperature is $84^{\circ} \mathrm{C}$.

Amplification reactions were performed in a final volume of $25 \mu \mathrm{L}$ with $1 \mathrm{X}$ SYBR Green I Amplification Master Mix (Euroclone, Milan, Italy), 150 picomoles of the forward primer, 50 picomoles of the reverse primer and $10 \mathrm{ng}$ of genomic DNA. The cycling conditions were as follows: $95^{\circ} \mathrm{C}$ for $10 \mathrm{~min}$ followed by $95^{\circ} \mathrm{C}$ for $30 \mathrm{~s}$ and $60^{\circ} \mathrm{C}$ for $1 \mathrm{~min}$, repeated 45 times in the Rotor Gene-3000 apparatus (Corbett Research Mortlake, Sydney, Australia). At the end of the PCR, the dissociation protocol included a slow heating from $60^{\circ}$ to $95^{\circ} \mathrm{C}$ in $0.2^{\circ} \mathrm{C}$ steps, with an 8-s interval between steps. Melting curve profiles were obtained using the dissociation software of the Rotor Gene3000 apparatus. Samples were analyzed in duplicate and in different PCR runs to test the reproducibility of this technique.

Allele frequencies were calculated by direct gene counting and the differences analyzed by the chi-square test using $2 \times 2$ contingency table.

Our melting temperature assay protocol permitted easy and rapid MBL2 genotyping of the 164 HIV-1-infected Brazilian patients and 150 healthy controls. As described by Garred et al. (11), the three MBL2 variants (Arg52Cys, Gly54Asp, Gly57Glu) were grouped together and called allele 0 , while the combination of three wild-type alleles was called allele A. Examples of melting profiles of the three MBL2 genotypes A/A, $\mathrm{A} / 0$ and $0 / 0$ are reported in Figure 1 . The melting temperatures of the three MBL2 genotypes were as follows: A/A (one peak of $83.1 \pm 0.1^{\circ} \mathrm{C}$ ), $\mathrm{A} / 0$ (two peaks of $82.6 \pm$ 0.3 and $80.7 \pm 0.1^{\circ} \mathrm{C}$ ), and $0 / 0$ (one peak of $\left.81.7 \pm 0.1^{\circ} \mathrm{C}\right)$.

MBL2 genotypes were obtained directly after the the melting protocol using the dissociation software of Rotor Gene-3000. No additional software was needed to analyze the raw data and the results were not imported to a new data sheet for a more accurate analysis as indicated by Hladnik et al. (10). Our protocol allowed fast MBL2 genotyping, requiring less than $3 \mathrm{~h}$.

The genotypes of 30 randomly chosen HIV-1-infected children and 30 healthy controls were confirmed by direct PCR sequencing of the first exon of the MBL2 gene, containing the three polymorphisms.

The Rotor Gene-3000 dissociation software permitted the automatic calling of MBL2 genotypes by inserting as "Bin" values the melting temperature assigned to each MBL2 genotype. Any other variation of the MBL2 amplicon sequence would be characterized by a different melting temperature, easily distinguishable from the known three allelic variants of MBL2. One of 30 healthy controls was characterized by the presence of an SNP different from the three known allelic variants $(\mathrm{G} \rightarrow \mathrm{A}$ at position 24 of the 90-bp amplicon) and both the melting temperature of the amplicon $\left(85^{\circ} \mathrm{C}\right)$ and the melting profile changed, being discarded by the automatic genotyping software of Rotor Gene-3000 (data not shown).

Samples characterized by the three MBL2 variants (Arg52Cys, Gly54Asp, Gly57Glu) always showed the same melting profile with the following standard deviation (SD) values for the wild-type homozygotes $(\mathrm{SD} \pm$ $0.1^{\circ} \mathrm{C}$ ), the wild-type/mutated heterozygotes $(\mathrm{SD} \pm 0.3)$ and the mutated/mutated homozygotes $\left(\mathrm{SD} \pm 0.1^{\circ} \mathrm{C}\right)$.

The frequencies of MBL2 polymorphisms in HIV-1-infected children and healthy controls are reported in Table 1. The frequency of the A/A homozygotes was significantly higher among healthy controls $(0.68)$ than in HIV-infected children $(0.55 ; \mathrm{P}=0.0234)$ and the frequency of MBL2 0/0 homozygotes was higher among HIV-1-infected children than healthy controls $(P=0.0296)$. The 0 allele was significantly more frequent among the 164 HIV-1-infected children 
Figure 1. Melting profile of the amplicon of MBL2 homozygous $\mathrm{A} / \mathrm{A}$ (one peak), heterozygous $\mathrm{A} /$ 0 (two peaks) and homozygous 0/0 (one peak) subjects obtained using the Rotor Gene-3000 dissociation curve software. The three genotypes are easily distinguishable.

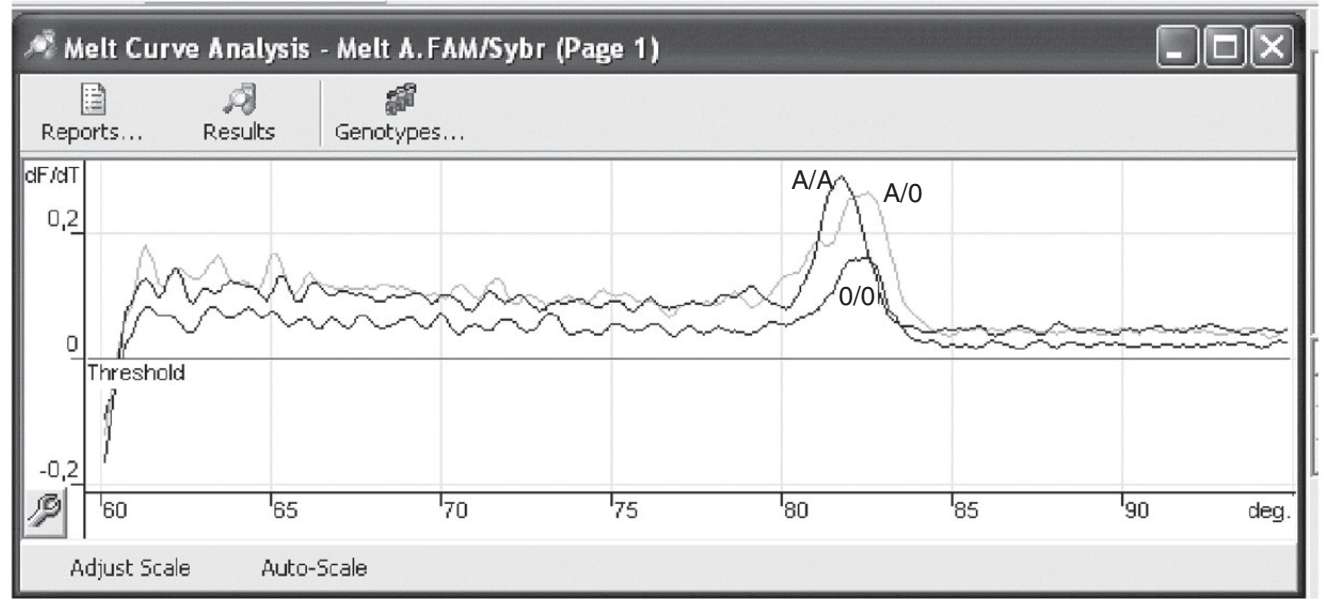

Table 1. Polymorphism frequencies at the first exon of the MBL2 gene in HIV-1-infected children and panethnic healthy controls.

HIV-1-infected children $(\mathrm{N}=164)$

Healthy controls $(N=150)$

MBL2 genotype frequencies

$\begin{array}{llc}\text { A } / A & 90 / 164(55 \%) & 102 / 150(68 \%)^{\star} \\ A / 0 & 54 / 164(33 \%) & 41 / 150(27 \%) \\ 0 / 0 & 20 / 164(12 \%)^{\star \star} & 7 / 150(5 \%)\end{array}$

MBL2 allele frequencies

$\begin{array}{lrr}\text { A } & 234 / 328(71 \%) & 245 / 300(82 \%) \\ 0 & 94 / 328(29 \%)^{+} & 55 / 300(18 \%)\end{array}$

Data are reported as number of patients (\% of total).

${ }^{*} \mathrm{P}=0.0234$ compared to HIV-1-infected children; ${ }^{* *} \mathrm{P}=0.0296$ compared to healthy controls. ${ }^{+} \mathrm{P}=0.0032$ compared to healthy controls (chi-square test using $2 \times 2$ contingency table).

(0.29) than among the 150 healthy controls (0.18; $\mathrm{P}=0.0032)$. Our data confirm the association between the presence of the mutated MBL2 allele (allele 0) and HIV-1 infection in perinatally exposed children, as reported by Boniotto et al. (12).

The MBL2 genotype frequencies obtained here were in agreement with HardyWeinberg equilibrium for the two cohorts analyzed, further confirming the reliability of the assay.

Recently, some new melting temperature assay protocols have been developed for real time PCR genotyping using SYBR Green I chemistry. Some of them require post-PCR manipulation (14), increasing the risk of cross contamination, while others $(10)$ require timeconsuming data handling procedures.
Our MBL2 genotyping melting temperature assay gave robust and reproducible results, characterized by very low SD and verified in blind by direct sequencing, without any manipulation after real time PCR and no supplementary data handling since the genotypes were correctly determined by the Rotor Gene-3000 dissociation protocol software. Moreover, the use of SYBR Green I chemistry instead of expensive fluorophorelabeled probes significantly reduced the cost of the assay.

We suggest the use of our SYBR Green I melting temperature assay protocol for rapid and reproducible MBL2 genotyping in laboratories simply equipped since the protocol only requires a real time PCR machine. 


\section{References}

1. Gray IC, Campbell DA, Spurr NK. Single nucleotide polymorphisms as tools in human genetics. Hum Mol Genet 2000; 9: 2403-2408.

2. Meyer K, Fredriksen A, Ueland PM. High-level multiplex genotyping of polymorphisms involved in folate or homocysteine metabolism by matrix-assisted laser desorption/ionization mass spectrometry. Clin Chem 2004; 50: 391-402.

3. Ou-Yang H, Hua L, Mo QH, Xu XM. Rapid, accurate genotyping of the common-alpha(4.2) thalassaemia deletion based on the use of denaturing HPLC. J Clin Pathol 2004; 57: 159-163.

4. Pirulli D, Giordano M, Puzzer D, Crovella S, Rigato I, Tiribelli C, et al. Rapid method for detection of extra (TA) in the promoter of the bilirubin-UDP-glucuronosyl transferase 1 gene associated with Gilbert syndrome. Clin Chem 2000; 46: 129-131.

5. Pirulli D, Giordano M, Lessi M, Spano A, Puzzer D, Zezlina S, et al. Detection of AGXT bgene mutations by denaturing high-performance liquid chromatography for diagnosis of hyperoxaluria type 1. Clin Exp Med 2001; 1: 99-104.

6. Flavell AJ, Bolshakov VN, Booth A, Jing R, Russell J, Ellis TH, et al. A microarray-based high throughput molecular marker genotyping method: the tagged microarray marker (TAM) approach. Nucleic Acids Res 2003; 31: e115.

7. Lee LG, Livak KJ, Mullah B, Graham RJ, Vinayak RS, Woudenberg TM. Seven-color, homogeneous detection of six PCR products.
Biotechniques 1999; 27: 342-349.

8. Tyagi S, Bratu DP, Kramer FR. Multicolor molecular beacons for allele discrimination. Nat Biotechnol 1998; 16: 49-53.

9. Ririe KM, Rasmussen RP, Wittwer CT. Product differentiation by analysis of DNA melting curves during the polymerase chain reaction. Anal Biochem 1997; 245: 154-160.

10. Hladnik U, Braida L, Boniotto M, Pirulli D, Gerin F, Amoroso A, et al. Single-tube genotyping of MBL-2 polymorphisms using melting temperature analysis. Clin Exp Med 2002; 2: 105-108.

11. Garred P, Madsen HO, Balslev U, Hofmann B, Pedersen C, Gerstoft $J$, et al. Susceptibility to HIV infection and progression of AIDS in relation to variant alleles of mannose-binding lectin. Lancet 1997; 349: 236-240.

12. Boniotto M, Braida L, Pirulli D, Arraes L, Amoroso A, Crovella S. MBL2 polymorphisms are involved in HIV-1 infection in Brazilian perinatally infected children. AIDS 2003; 17: 779-780.

13. Amoroso A, Berrino M, Boniotto M, Crovella S, Palomba E, Scarlatti $\mathrm{G}$, et al. Polymorphism at codon 54 of mannose-binding protein gene influences AIDS progression but not HIV infection in exposed children. AIDS 1999; 13: 863-864.

14. Lipsky RH, Mazzanti CM, Rudolph JG, Xu K, Vyas G, Bozak D, et al. DNA melting analysis for detection of single nucleotide polymorphisms. Clin Chem 2001; 47: 635-644. 